\title{
Application of an evolutionary algorithm in the optimal design of micro-sensor ${ }^{1}$
}

\author{
Qibing Lu ${ }^{\mathrm{a},{ }^{*}}$, Pan Wang ${ }^{\mathrm{b},{ }^{*}}$, Sihai Guo ${ }^{\mathrm{b}, *}$, Buyun Sheng ${ }^{\mathrm{a}}$, Xingxing Liu ${ }^{\mathrm{b}}$ and Zhun Fan ${ }^{\mathrm{a}, \mathrm{c}}$ \\ ${ }^{a}$ Hubei Digital Manufacturing Key Laboratory, School of Mechanical and Electrical Engineering, \\ Wuhan University of Technology, Wuhan, China \\ ${ }^{b}$ Institute of Systems Science and Engineering, Wuhan University of Technology, Wuhan, China \\ ${ }^{c}$ School of Engineering, Shantou University, Shantou, China
}

\begin{abstract}
This paper introduces an automatic bond graph design method based on genetic programming for the evolutionary design of micro-resonator. First, the system-level behavioral model is discussed, which based on genetic programming and bond graph. Then, the geometry parameters of components are automatically optimized, by using the genetic algorithm with constraints. To illustrate this approach, a typical device micro-resonator is designed as an example in biomedicine. This paper provides a new idea for the automatic optimization design of biomedical sensors by evolutionary calculation.
\end{abstract}

Keywords: Genetic programming (GP), bond graph (BG), micro-resonator, evolutionary computation

\section{Introduction}

With the continuous development of the micro electro mechanical systems (MEMS) technology and biological chip technology, the concept of the current biosensor has jumped out of the original narrow circle. It has extended to miniaturization, integration, intelligent and chip for characteristics of micro system for biological detection and treatment. Optimization design of biosensor also affects the development of biomedicine.

At present, there have been many reports on research of biological micro-sensor based on MEMS process. The study of enzyme sensor is mature, the DNA sensor research is continuous, and the immune sensor research is still at the start stage. The signal converters are the most typical applications, such as biological micro-sensor, micro cantilever, microelectrode, micro bulk acoustic wave resonator and biological sensitive field effect transistor.

\footnotetext{
${ }^{1}$ This study was supported by National Natural Science Foundation of China (No. 71371148, 61175073).

* Address for correspondence: Qibing Lu, Hubei Digital Manufacturing Key Laboratory, School of Mechanical and Electrical Engineering, Wuhan University of Technology, Wuhan, China. Tel.: +86 13429816020; Fax: +86-27-87859049; E-mail: luqibing1988@163.com.

Pan Wang, Institute of Systems Science and Engineering, Wuhan University of Technology, Wuhan, China. Tel.: +86-2787858435; Fax: +86-27-87859049; E-mail: wangpan@whut.edu.cn.

Sihai Guo, Institute of Systems Science and Engineering, Wuhan University of Technology, Wuhan, China. Tel.: +86-2787858435; Fax:+86-27-87859049; E-mail: guosihai@whut.edu.cn.
} 
MEMS is an extremely complex system effected by the multi physics field coupling. Most simulation tools confined to just one energy field, so it needs a new design methodology to improve the design of such systems [1]. This paper introduces an automatic bond graph design method based on genetic programming for the evolutionary design of micro-resonator. In addition to keeping the advantages of bond graph, a new design method is integrated in the introduced method. To realize automatic design through evolutionary calculation, a new idea is provided for the design of complex multi-domain systems. According to the micro electro mechanical systems (MEMS) classification proposed by Senturia [2], the interactive modeling and simulation of MEMS devices and electronic devices could be realized at the system level. Bond Graph (BG) [3], as a modeling method, provides a unified approach for the modeling and analysis of dynamic systems. Meanwhile, genetic programming (GP) [4] is a kind of evolutionary algorithms that involve a typical tree structure. Experimental data shows that after defining the feasibility function set, genetic programming (GP) could also evolve the corresponding RF MEM devices, i.e. the topology and parameters of band pass filter, can be evolutionarily improved through genetic programming, so as to satisfy predefined design specifications. The advantage of the proposed method lies in its open search capacity in topology design space and parameter design capacity. The method employs bond graph to represent the models of mechanical and electrical products, and genetic programming to design space search. There are some successful examples of using genetic programming for the modeling and automatic synthesis of physical systems [5-7], but the search spaces of the topologies in those examples are greatly limited. The method proposed in this paper overcomes such limitation by its (1) Analysis of multi-energy and multi-domain systems by using bond graph; (2) Open search of design topology structures by using genetic programming [8]; and (3) Introduction of an effective and fast multi-step evaluation for genetic programming based on the characteristics of bond graph.

\section{Hierarchy-based MEMS design methods}

With the development of computer technology, new intellective heuristic algorithms are emerging and being applied broadly [9-11]. The research on automatic MEMS design methods is both a new challenge and a successor to previous studies [12]. MEMS design process is divided into system-level design, device-level design and process-level design, each has its own design features and design goals, as well as its own design and optimization methods. According to the order in which the three-tier design proceeds, MEMS design methods can be classified into top-down design and bottom-up design approaches. A top-down design process can be verified by using a bottom-up method to see whether the design of each level strictly meets the requirements. The GP/BG method is easily extended and applied to the system-level MEMS design. Design issues of the physical layer are expressed as constrained optimization problems and solved using a constrained evolutionary algorithm [13].

GP / BG method adopts genetic programming method to study the modification of design space and the advantages of bond graph, in order to more accurately and efficiently model multi-domain systems, proving its feasibility in the integrated design of multi-domain dynamic systems [14, 15]. In the firstlevel or advanced MEMS design, GP / BG method can help obtain a high-level description. This system puts together a collection of existing component libraries, through an automated way, to meet predefined design specifications. For the second- or lower-level design, other digital optimization methods [16] are used for evolutionary computation in order to synthesize custom components that meet functional specifications. Figure 1 illustrates the structural design process of MEMS. The GP /BG method aims to solve system-level design issues at the first level in an automated manner. 


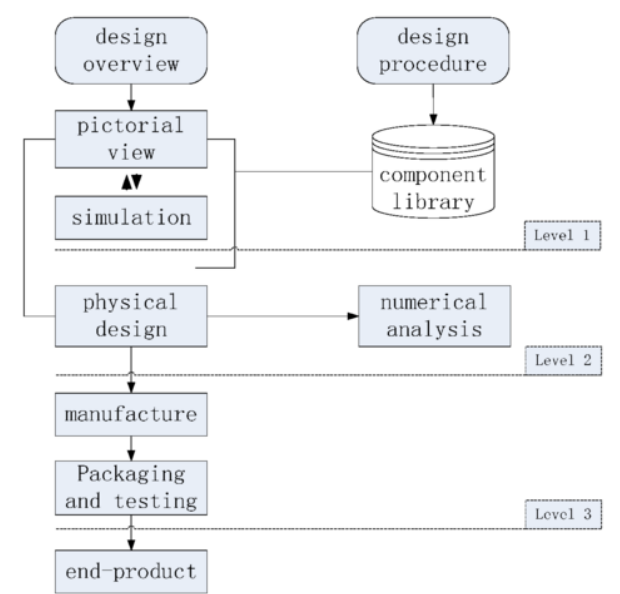

Fig. 1. Structure design process based on micro electro mechanical system.

\section{Evolutionary design based on bond graph and genetic programming}

Genetic algorithm-based designs are only applicable to the design of search space with fixed size of design solution. However, in open innovative design of bond graph, the size of the design solution is uncertain; the number of components, the way of connection, and the component parameter setup are not given in advance. The breakthrough idea of genetic programming-based evolutionary design is to realize design space search through evolutionarily elongating program instead of direct programming. The process of bond graph-based evolutionary design is detailed below [17].

(1) The generation of evolutionary population: The target of search is a set of candidates, rather than an individual design.

(2) The design of embryo bond graphs: Prior to the evolutionary design, the designer must provide the embryo bond graph $[18,19]$. Figure 2 presents an embryo bond graph modifiable points; the dashed box illustrates the initial modifiable points.

(3) The design of the topological operator of genetic programming function sets: The GP function sets are the components that modify the structure and parameters. There are four types of GP functions: add, insert, replace and numerical function.

(4) The crossover and mutation operations of the design: through genetic manipulation functions, the structure of GP trees can be modified, so as to realize individual evolution, as shown in Figure 3.

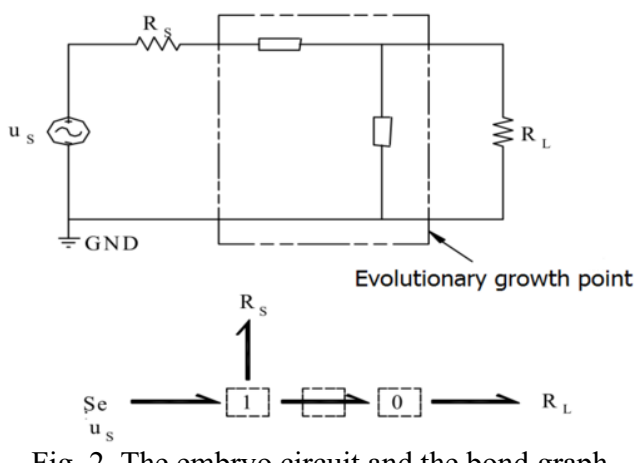

Fig. 2. The embryo circuit and the bond graph. 


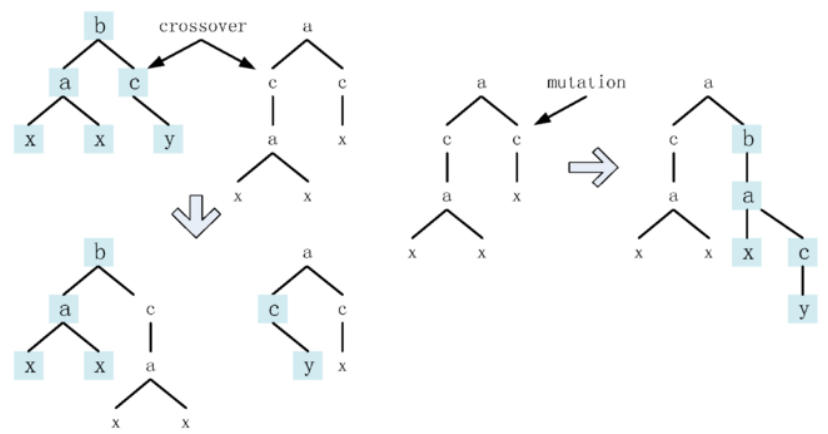

Fig. 3. GP genetic operator based on BG.

(5) The assessment of fitness value: For a given bond graph model, its fitness value is assessed with the following steps:

(1) obtain matrix A of the state space model from the bond graph model;

(2) calculate the eigenvalues of matrix A;

(3) compare the eigenvalues of the calculation results and the objective eigenvalues, and calculate the D-value; in the range of 0 to 1 , calculate the fitness value of bond graph by the following rule:

if distance/order $<0.1$ then

fitness $=0.1 /(0.1+$ distance $/$ order $)$

else fitness $=5.05 /(10+$ distance $/$ order $)$

where "order" is the value of the energy storage element.

(6) The selection operation: the units with higher fitness values are more likely to be selected to perform a copy operation. The principle for selection is "survival of the fittest".

This paper introduces the realization of the automatic design of bond graph through evolutionary algorithm, and the dynamic design of multi-domain systems. Random selection, crossover, and mutation operator guide the evolutionary design process. The evolutionary process has to be stopped when the fitness value has reached a specified value or the desired bond graph has been found. Multiple candidate solutions with the highest fitness values should be output as the result of bond graph design.

\section{Case study: comb-driven microresonator design}

A comb-drive microresonator design with 15 mixed-type design variables and 24-design constraints is proposed in this paper. The comb-drive microresonator is fabricated in a poly-silicon surface micro structural process [20]. The layout of the comb-driven microresonator is shown in Figure 4(a). It can be specified by 15-design variables, as shown in Figure 4(b). The vector of design variables are defined as follows:

$$
\vec{x}=\left[L_{b}, \omega_{b}, L_{t}, \omega_{t}, L_{s y}, \omega_{s y}, \omega_{s a}, \omega_{c y}, L_{c y}, L_{c}, \omega_{c}, L_{s a}, x_{o}, V, N_{c}\right]
$$

Units of micrometers are noted for the first 13-design variables. They should be integer multiples of the feature size (set to be $0.09 \mu \mathrm{m}$ ), then they are discrete variables. The 14th-design variable is a continuous variable with unit of volt. The 15th-design variable is an integer variable with no unit. 15 
Table 1

15 mixed-type design variables for microresonator

\begin{tabular}{|l|l|l|l|l|l|l|l|}
\hline Design Variable & Design constraints & Design Variable & \multicolumn{2}{l|}{ Design constraints } \\
\hline Variable & Description & Min & Max & Variable & Description & Min & Max \\
\hline$L_{b}$ & length of flexure beam & 2 & 400 & $L_{c}$ & length of comb fingers & 8 & 400 \\
\hline$\omega_{b}$ & width of flexure beam & 2 & 20 & $\omega_{c}$ & width of comb fingers & 2 & 20 \\
\hline$L_{t}$ & length of truss beam & 2 & 400 & $\omega_{s a}$ & width of shuttle axle & 10 & 400 \\
\hline$\omega_{t}$ & width of truss beam & 2 & 20 & $L_{s a}$ & length of shuttle axle & 2 & 400 \\
\hline$L_{s y}$ & length of shuttle yoke & 2 & 400 & $x_{o}$ & comb finger overlap & 4 & 400 \\
\hline$\omega_{s y}$ & width of shuttle yoke & 10 & 400 & V & voltage amplitude & 0 & 50 \\
\hline$L_{c y}$ & length of comb yoke & 2 & 700 & $N_{c}$ & number of rotor comb fingers & 3 & 50 \\
\hline$\omega_{c y}$ & width of comb yoke & 10 & 400 & & & & \\
\hline
\end{tabular}

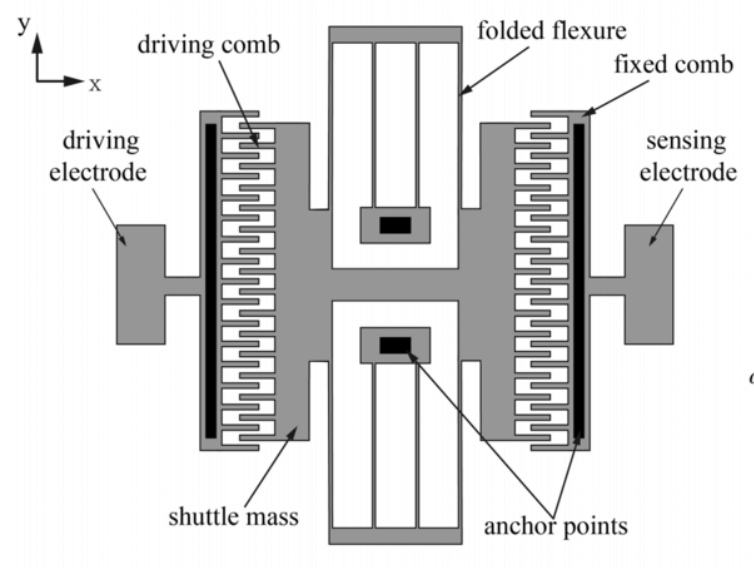

(a)

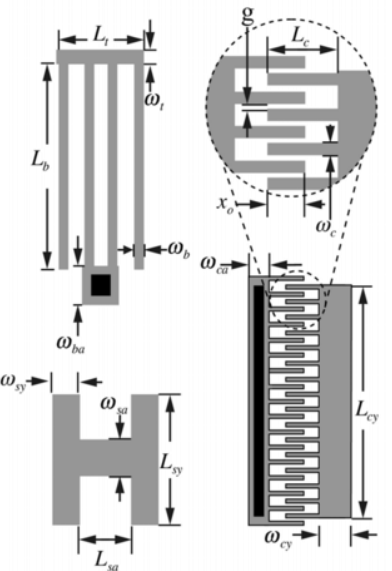

(b)

Fig. 4. (a) Comb-drive microresonator fabricated in a poly-silicon surface micro structural process. (b) Major design variables for comb-driven microresonator.

mixed-type design variables for microresonator are shown in Table 1.

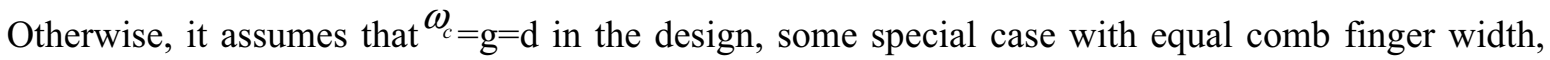
gap, and spacing. $\omega_{b a}=11, \omega_{c a}=14, \quad \gamma=4, \mathrm{t}=2$, in which $\omega_{b a}$ is the width of beam anchors, $\omega_{c a}$ is the width of stator comb, and $t$ is the thickness of the microresonator.

Some design constraints, both geometric and functional constraints, are considered for the combdriven microresonator cell component. In this paper, the following 12 constraints are considered [21]:

$$
\begin{array}{ll}
g_{1}(x): 0 \leq 2 \omega_{c}+2 g+L_{c y} \leq 700 & g_{5}(x): 0 \leq\left(2 N_{c}+1\right) W_{c}-L_{c y}+2 N_{c} g \leq 700 \\
g_{2}(x): 0 \leq 2 \omega_{t}+2 L_{b}+L_{s y} \leq 700 & g_{6}(x): 4 \leq x_{0}-x_{d i s p} \leq 200 \\
g_{3}(x): 0 \leq 2 \omega_{c y}-2 x_{0}+4 L_{c}+\omega_{s y}+2 \omega_{c a}+3 L_{t} \leq 700 & g_{7}(x): 4 \leq L_{t}-\left(W_{s y}+W_{b}\right) / 2-x_{d i s p} \leq 200 \\
g_{4}(x): 4 \leq L_{c}-\left(x_{d i s p}+x_{0}\right) \leq 200 & g_{8}(x): 4 \leq L_{s y}-W_{s a}-2 W_{b a} \leq 400
\end{array}
$$


$g_{9}(x): 2 \leq x_{\text {disp }} \leq 100$
$g_{10}(x): 5 \leq Q \leq 1 e 5$

$$
\begin{aligned}
& g_{11}(x): 0 \leq x_{\text {disp }} / L_{b} \leq 0.1 \\
& g_{12}(x): 0 \leq K_{e, y} / K_{y} \leq 1 / 3
\end{aligned}
$$

The first 8-design constraints are linear, and the last 4-design constraints are nonlinear. $x_{d i s p}=Q F_{e, x} / K_{x}$, where $\mathrm{Q}$ is the quality factor: $Q=\sqrt{m_{x} K_{x} / B_{x}{ }^{2}}, F_{e, x}$ is the force generated by the comb drive. The force is proportional to the square of the voltage $\mathrm{V}$ applied across the comb fingers. $F_{e, x}=1.12 \varepsilon_{0} N_{c} \frac{t}{g} V^{2}$, where $\varepsilon_{0}$ is the permittivity of air. $K_{x}=\frac{2 E t W_{b}{ }^{3}}{L_{b}{ }^{3}} \frac{L_{t}{ }^{2}+14 \alpha L_{t} L_{b}+36 \alpha^{2} L_{b}{ }^{2}}{4 L_{t}{ }^{2}+41 \alpha L_{t} L_{b}+36 \alpha^{2} L_{b}{ }^{2}}$, where $B_{x}=\mu\left[\left(A_{s}+0.5 A_{t}+0.5 A_{b}\right)\left(\frac{1}{d}+\frac{1}{\gamma}\right)+\frac{A_{c}}{g}\right], \alpha=\left(W_{t} / W_{b}\right)^{3}$, where $A_{b}, A_{c}, A_{s}$ and $A_{t}$, are flexure beams, comb finger sidewalls, the bloated layout areas of the shuttle, and truss beams, respectively. $\mu$ is air viscosity. Moreover, $m_{x}=m_{s}+\frac{1}{4} m_{t}+\frac{12}{35} m_{b}$, where $m_{s}=\rho A_{s} t, m_{t}=\rho A_{t} t, m_{b}=\rho A_{b} t . A_{s}=\omega_{s a} L_{s a}+2 \omega_{s y} L_{s y}$, $A_{t}=2 \omega_{c a} L_{c y}, A_{b}=8 \omega_{b} L_{b}+2 \omega_{t}\left(2 L_{t}+\omega_{a}+2 \omega_{b}\right), A_{c}=2 N_{c} \omega_{c} L_{c}$. The natural frequency $\omega_{n}: \omega_{n}=\frac{1}{2 \pi} \sqrt{\frac{K_{x}}{m_{x}}}$. The design objective of comb-driven microresonator is robustly to match the natural frequency with a predefined natural frequency.

In this paper, a genetic algorithm with constraints is used to provide a search orientation towards the feasible domain. Once the feasible solutions are found, the niche algorithm is used to keep the diversity of the feasible solution. It makes the crossover operator of the genetic algorithm to find better feasible solution, make the search close to the true optimal solution.

The parameter setting of the genetic algorithm with constraints as shown in Table 2, and flow chart of the proposed algorithm is shown in Figure 5.

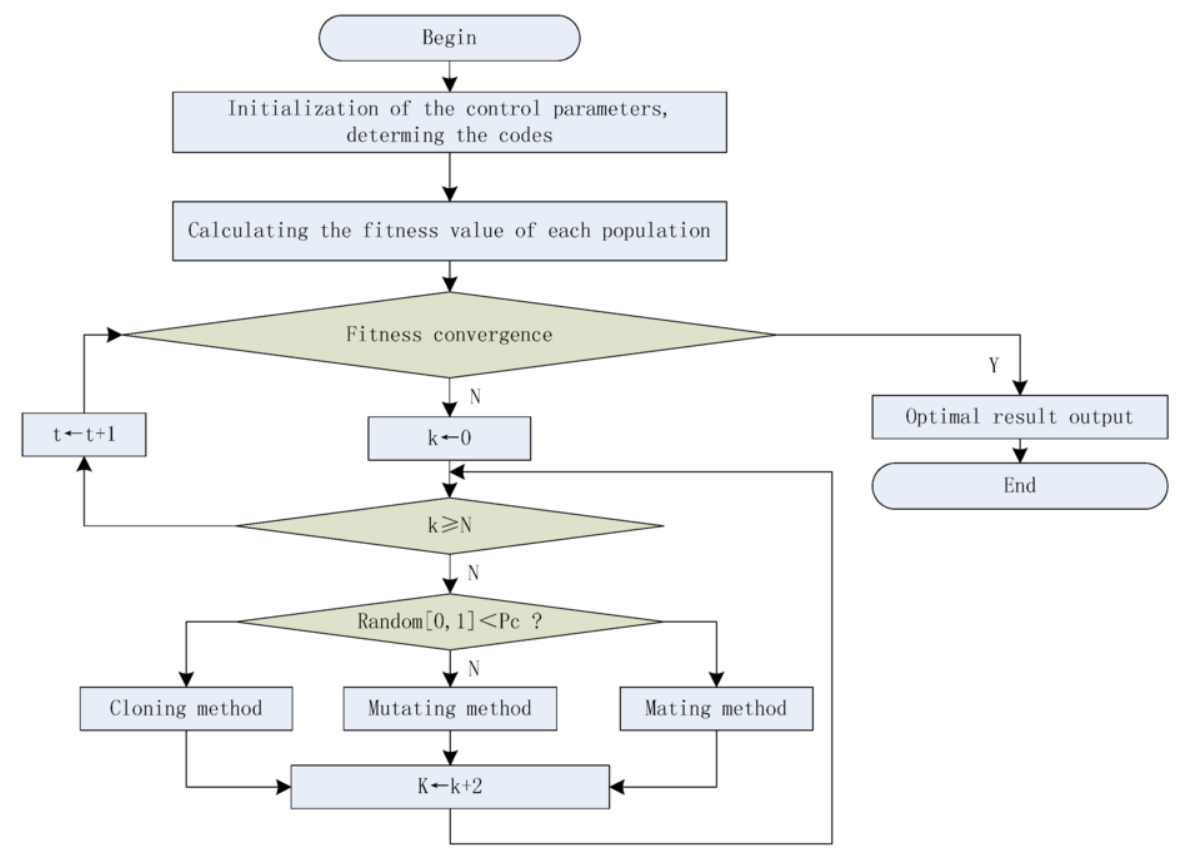

Fig. 5. Flow chart of the proposed algorithm. 
Table 2

The parameter of the genetic algorithm with constraints

\begin{tabular}{|l|l|}
\hline variable boundary: Strict & population quantity: 500 \\
\hline total generation count: 100 & Crossover probability: 0.9 \\
\hline mutation probability: 0.15 & niche Count: 0.9 \\
\hline n for SBX: 2 & n for mutation: 50 \\
\hline
\end{tabular}

Table 3

Layout parameters after ten runs by the genetic algorithm

\begin{tabular}{|l|l|l|l|l|l|l|l|l|l|l|}
\hline RUN NO. & 1 & 2 & 3 & 4 & 5 & 6 & 7 & 8 & 9 & 10 \\
\hline$L_{b}(\mu \mathrm{m})$ & 308.63 & 306.44 & 307.35 & 308.72 & 306.24 & 312.07 & 307.47 & 308.88 & 310.26 & 306.61 \\
\hline$\omega_{b}(\mu \mathrm{m})$ & 17.82 & 17.82 & 17.79 & 17.81 & 17.80 & 17.80 & 17.80 & 17.79 & 17.79 & 17.80 \\
\hline$L_{t}(\mu \mathrm{m})$ & 183.26 & 195.28 & 136.84 & 122.37 & 168.13 & 172.09 & 189.67 & 162.79 & 135.57 & 203.72 \\
\hline$\omega_{t}(\mu \mathrm{m})$ & 2.08 & 3.49 & 2.09 & 2.31 & 1.95 & 1.99 & 2.88 & 3.24 & 2.07 & 2.68 \\
\hline$L_{s y}(\mu \mathrm{m})$ & 58.65 & 67.28 & 38.97 & 48.34 & 64.12 & 95.83 & 107.21 & 69.88 & 73.36 & 87.55 \\
\hline$\omega_{s y}(\mu \mathrm{m})$ & 18.14 & 12.53 & 15.78 & 10.24 & 11.70 & 11.53 & 15.62 & 19.17 & 16.30 & 18.70 \\
\hline$\omega_{s a}(\mu \mathrm{m})$ & 10.80 & 11.28 & 15.59 & 13.12 & 10.87 & 11.30 & 18.26 & 17.69 & 10.44 & 11.18 \\
\hline$\omega_{c y}(\mu \mathrm{m})$ & 12.15 & 10.17 & 10.09 & 12.81 & 10.24 & 14.79 & 23.80 & 17.91 & 11.63 & 10.47 \\
\hline$L_{c y}(\mu \mathrm{m})$ & 578.92 & 643.28 & 648.71 & 664.17 & 651.88 & 631.08 & 396.47 & 617.29 & 649.73 & 641.91 \\
\hline$L_{c}(\mu \mathrm{m})$ & 12.03 & 12.02 & 12.02 & 12.02 & 12.02 & 12.06 & 12.18 & 16.30 & 12.11 & 12.06 \\
\hline$\omega_{c}(\mu \mathrm{m})$ & 9.68 & 9.82 & 10.08 & 12.37 & 9.32 & 11.59 & 10.32 & 10.23 & 12.18 & 9.26 \\
\hline$L_{s a}(\mu \mathrm{m})$ & 382.08 & 371.57 & 294.64 & 103.33 & 20.27 & 25.42 & 93.67 & 13.71 & 12.79 & 227.63 \\
\hline$x_{0}(\mu \mathrm{m})$ & 6.21 & 6.21 & 6.21 & 6.23 & 6.23 & 6.30 & 18.98 & 6.24 & 6.18 & 6.20 \\
\hline$V($ volt $)$ & 32 & 35 & 36 & 33 & 28 & 37 & 36 & 27 & 26 & 33 \\
\hline Obj. value & $1 \mathrm{e}-6$ & $4 \mathrm{e}-6$ & $3 \mathrm{e}-6$ & $1 \mathrm{e}-6$ & $2 \mathrm{e}-6$ & $1 \mathrm{e}-6$ & $1.5 \mathrm{e}-5$ & $1 \mathrm{e}-6$ & $3 \mathrm{e}-6$ & $2 \mathrm{e}-6$ \\
\hline
\end{tabular}

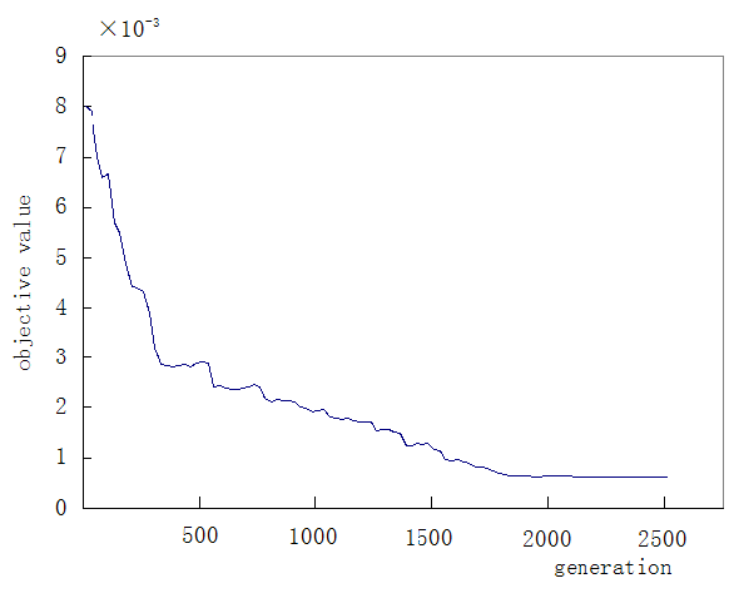

Fig. 6. SSE-Generation curve graphs.

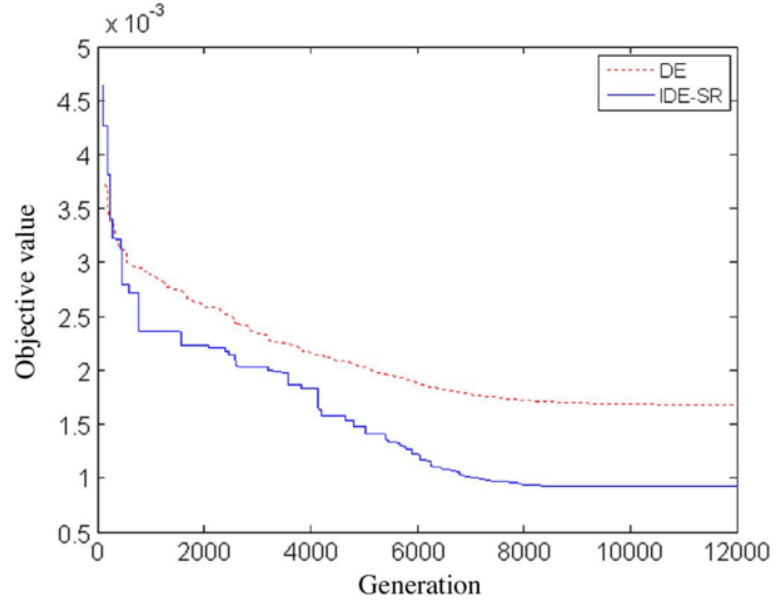

Fig. 7. Curves of objective value versus generation of both DE and IDE-SR. 
After running ten times genetic algorithm by using different random seeds, the parameter and objective function value with adjustable size are obtained, as shown in Table 3 . As can be seen, the performance of the algorithm is very stable. Almost all of the operation has obtained the target value, the range of normalized squared sum of errors is $1.0 \mathrm{e}-6$. The average value is $3.5 \mathrm{e}-6$, the standard deviation is $3.8533 \mathrm{e}-6$, the maximum value of NSSE is $1.5 \mathrm{e}-5$, and it can be regarded as superb results. Figure 6 is a typical SSE-Generation curve, and it can be seen from the chart, according to the changes of generations, the NSSE value reduced to close to linear, in the 1800-th generation, the NSSE value is reduced to $1.0 \mathrm{e}-6$.

Figure 7 shows the curves of objective values versus generation number recorded in one exemplar evolutionary process of both standard DE and IDE-SR. [20] Comparing Figure 6 and Figure 7, it shows that after 1600 iterations, the NSSE value has reduced to below 1.0e-3 and tended to be stable, this algorithm has faster convergence speed and relatively lower NSSE value, the validity and convergence of the algorithm are better than IDE-SR and standard DE.

\section{Conclusion}

A hierarchical design method for MEMS automatic synthesis is put forward in this paper. It is the first time for MEMS automatic synthesis process hierarchy in a comprehensive framework. First, the system-level behavioral model is discussed, which based on genetic programming and bond graph. Then, the geometry parameters of components are automatically optimized, by using the genetic algorithm with constraints. To illustrate this approach, a typical device micro-resonator is designed as an example in biomedicine. In addition, it still need further investigation to optimize multiple design goals of the system, in order to reproduce offspring to meeting the ideal design, so that the various subsystems and components meet the design specification. Meanwhile, the multi-objective fitness function in evolvable systems is valuable to be used into the issue of MEMS design [22].

\section{References}

[1] Z. Fan, K. Seo, J. Hu, et al., A novel evolutionary engineering design approach for mixed-domain systems, Journal of Engineering Optimization 36 (2004), 127-147.

[2] S.D. Senturia, Microsystem Design, Kluwer Academic Publishers, Dordrecht, 2001

[3] Z.S. Wang, Application of Bond Graph Theory and Its Application in System Dynamics, Harbin Engineering University Press, Harbin, 2007.

[4] J.R. Koza, Genetic Programming: On the Programming of Computers by Means of Natural Selection, MIT Press, Cambridge, 1992.

[5] J.J. Hu, E. Goodman and R. Rosenberg, Topologically opened ended synthesis of dynamic systems with high robustness using genetic programming: A case study of analog filter synthesis, IEEE Transactions on Evolutionary Computation, 2005, 1619-1626.

[6] S.B. Li and J.J. Hu, Automated design of mechatronic system based on genetic programming \& bond graphs, Journal of System Simulation 11 (2002), 1513-1516.

[7] S.B. Li and J.J. Hu, Evolving vibration absorbers based on genetic programming and bond graphs, Proceedings of the 2006 International Conference on Computational Intelligence and Security (CIS'2006) 10 (2006), 202-207.

[8] J. Hu, E. Goodman and R. Rosenberg, Topological search in automated mechatronic system synthesis using bond graphs and genetic programming, In Proc. of American Control Conference ACC 2004, Boston, 2004, 5628-5634.

[9] Tanja Macheiner, Anna Kuess, et al., A novel method for isolation of epithelial cells from ovine esophagus for tissue engineering, Bio-Medical Materials and Engineering 24 (2014), 1457-1468.

[10] W.P. Chen, Che-Lun Hung, et al., Novel and efficient tag SNPs selection algorithms, Bio-Medical Materials and Engineering 24 (2014), 1383-1389. 
[11] X. Jie, R. Cao and L. Li, Emotion recognition based on the sample entropy of EEG, Bio-Medical Materials and Engineering 24 (2014), 1185-1192.

[12] G.K. Fedder and Q. Jing, A hierarchical circuit-level design methodology for microelectromechanical system, IEEE Transactions on Circuits and Systems (TCAS) 46 (1999), 1309-1315.

[13] K. Deb, Efficient constraint handling method for genetic algorithms, Computer Methods in Applied Mechanics and Engineering 186 (2000), 311-338.

[14] Z. Fan, K. Seo, R.C. Rosenberg, et al., Exploring multiple design topologies using genetic programming and bond graphs, Proceedings of the Genetic and Evolutionary Computation Conference, GECCO-2002, New York, 2002, $1073-1080$.

[15] Z. Fan, J. Hu, K. Seo, et al., Bond graph representation and GP for automated analog filter design, Genetic and Evolutionary Computation Conference Late-Breaking Papers, San Francisco, 2001, 81-86.

[16] Y. Zhou, Layout Synthesis of Accelerometers, Thesis for Master of Science, Department of Electrical and computer Engineering, Carnegie Mellon University, 1998.

[17] Z. Fan, E.D. Goodman, J. Wang, et al., Hierarchical evolutionary synthesis of Mems, Proceedings of the Congress on Evolutionary Computation 2 (2004), 2320-2327.

[18] W.H. Di, B. Sun and L.X. Xu, Dynamic simulations of nonlinear multi-domain systems based on Genetic programming and bond graphs, Tsinghua Science and Technology 14 (2009), 612-616.

[19] Moin Lubna, Uddin Vali and Ali Shahid, System response analysis and model order reduction, using conventional method, bond graph technique and genetic programming, WMSCI 2008-The 12th World Multi-Conference on Systemics, Cybernetics and Informatics, Jointly with the 14th International Conference on Information Systems Analysis and Synthesis, ISAS 2008 - Proc. 3 (2008), 180-185.

[20] Z. Fan, J.C. Liu, Sorensen Torben and P. Wang, Improved differential evolution based on stochastic ranking for robust layout synthesis of MEMS components, IEEE Transactions on Industrial Electronics 56 (2009), 937-948.

[21] Z. Fan, J. Wang and E. Goodman, An evolutionary approach for robust layout synthesis of MEMS, IEEE/ASME International Conference on Advanced Intelligent Mechatronics, AIM 2 (2005), 1186-1191.

[22] P. Wang, J.J. Zhang, L. Xu, H. Wang, S. Feng and H.S. Zhu, How to measure adaptation complexity in evolvable systems-A new synthetic approach of constructing fitness functions, Expert Systems with Applications 38 (2011), 10414-10419. 